\title{
2021 Virtual MRS Spring Meeting \& Exhibit provided the latest in cutting-edge research
}

\author{
mrsmeetingscene.org
}

By Judy Meiksin, Arun Kumar, Jessalyn Hui Ying Low, and Victor A. Rodriguez-Toro

$\mathrm{O}$ nce again, due to caution during the COVID-19 pandemic, the Materials Research Society (MRS) held its Spring Meeting virtually on April 17-23, 2021, with recorded talks available through May 31, 2021. The 2021 Virtual MRS Spring Meeting \& Exhibit was chaired by Linyou Cao, the president of Atomix Inc.; Seung Min Jane Han, associate professor at the Korea Advanced Institute of Science and Technology; Lena F. Kourkoutis, associate professor at Cornell University; Andreas Lendlein, director of the Institute of Biomaterials Science, Helmholtz-Zentrum Hereon and professor at the University of Potsdam; and Xiaolin Li, a senior scientist and team lead of the battery development and reliability group at Pacific Northwest National Laboratory.

\section{Influence of the COVID-19 pandemic on the materials research community}

Since the World Health Organization declared the pandemic in March 2020, the emergency continues to be discussed in various ways at MRS Meetings, from the Science as Art competition, to the panel presentation organized by the MRS Women in Materials Science and Engineering Working Group, to the research reported in some of the symposia. Anna Salvati, an associate professor in the Groningen Research Institute of Pharmacy at the University of Groningen, The Netherlands, presented her latest results on nanomedicine (Symposium SM04), using corona molecules as an example.

For intracellular delivery of nanomedicines, nanomedicines have to first interact with the cell membrane and be recognized, after which they can be internalized by cells through a variety of cellular pathways. In her talk, Salvati explained the research work done to understand such interactions more deeply, and in particular how corona molecules can affect the internalization of drugs. "By understanding these interactions better, we can design nanomedicines to achieve the desired outcomes at cell level and control these interactions," said Salvati.

Salvati stated that when in a biological environment, a corona forms on the nanocarriers due to adsorption of surrounding biomolecules. This corona can be recognized by cell receptors, mediating the interaction between the cells and nanoparticles. It was found that for the same nanoparticle, but with different corona compositions, the internalization pathway of the nanoparticle also changes, implying that different corona compositions are recognized differently by cell receptors. Salvati also highlighted that even if a specific receptor is targeted, cells can internalize the nanoparticles through a different pathway as compared to the endogenous ligands. In addition, by changing the compositions of liposomes, the corona composition could be tuned - this in turn affects the kinetics and mechanisms of uptake by the cell.

To better understand the mechanisms of internalization, it is important to have superior models beyond conventional in vitro cell cultures. One such model developed by the research group are in vitro endothelial cell barriers that mimic the barriers that nanomedicines face in vivo. It was found that when cells developed into a barrier, endocytic markers were expressed to different levels, and had a lower nanoparticle uptake as compared to standard cell cultures. This indicates that the organization of the cells influences how nanoparticles are processed. Another model comprises precision-cut tissue slices-it was shown that precision-cut liver slices reproduced the preferential accumulation of nanoparticles by Kupffer cells as observed in vivo. This highlights how these models can be leveraged to better understand cellular internalization of nanomedicines occurring in vivo and optimize the design of nanomedicines.

During the COVID-19 pandemic, numerous reports have explored the particular difficulties women in academia have been experiencing. Surprisingly, the panelists in the Women in Materials Science \& Engineering Keynote Presentation: Perspectives and Take-Aways from the Global Pandemic provided tremendously optimistic views. This began with opening comments by Joanne Etheridge of Monash University in Australia when she said, "I think it's science that is guiding a path for us to humanity after this whole pandemic." She emphasized the expertise of scientists that has gotten the world through the pandemic, and that invented, designed, and developed vaccines within 12 months. "What a triumph of human achievement," she said. "It motivates me actually to be a scientist."

Payel Chatterjee at the Norwegian University of Science and Technology agreed: "Science can save humanity, 
nothing else can." The amount of misinformation and pseudoscience, especially in social media, encouraged Chatterjee to go further into public outreach. "I felt it's kind of part of my duty as a scientist to make people aware of what's actually going on in the lab," she said.

Sandra Young of the US Army DEVCOM remarked on the need for scientists to influence leadership. Before the pandemic, she said, researchers stayed in their laboratories and left it up to leadership to "pick and choose what they're going to highlight." What she learned from the pandemic is that scientists need to take responsibility to communicate the impact of their own work.

The panelists also discussed, of course, the difficulties of carrying on their work during the pandemic, while also balancing the needs at home and their own health and lifestyle changes. Everyone found a way. Colleagues stepped in as "family" checking in on one another's well-being, technology found its way to help keep families connected on the other side of the world. The moderator, Rebecca Anthony of Michigan State University, described the way she deliberately divided up time. Instead of succumbing to the kind of perpetual sort of super work - very pervasive in the US, she said - the pandemic led her to enforce boundaries for different activities.

Mmantsae Diale, a professor at the University of Pretoria in South Africa, wrapped up the session with even more positives. While technology may be talked about in terms of disrupting the status quo, "this time it is COVID-19 that forces you to use technology to your advantage," she said. Because of technology, Diale was able to continue working with her PhD students. "I was able to teach them anytime," she said, "because they can connect with me through WhatsApp and ask a question." Everyone learned well how to use the various communication platforms available, and to carefully plan what they can accomplish in a fixed period of time due to travel restrictions. Another great platform, Diale pointed out, is the networking platform at the virtual MRS Meeting. "Don't miss the opportunity to chat with us one-on-one," she said.

Graduate students Alekha Tyagi of the Indian Institute of Technology and Poorani Gnanasambandan of Luxembourg Institute of Science and Technology also had the pandemic on their minds when participating in the Science as Art competition. Both won first place in the popular vote by Meeting attendees who, apparently, were inspired by the images and their context (see Figures 1 and 2). Indirectly, the image by Sara Coppola, a research scientist at the National Research Council of Italy, Institute of Applied Sciences and Intelligent Systems (CNR ISASI), also gives a nod to this global concern, via an image of microneedles utilized as a drug delivery system (see Figure 3).

The remaining first and second place winning entries encompass other prominent areas of materials research. Graduate student Eunjin Choi of the Max Planck Institute for Intelligent Systems created art based on her work on nanodimers (see Figure 4); and Yadong Yin, a professor of chemistry at the University of California, Riverside, sent in an image inspired by his group's work on silicate nanosheet assemblies (see Figure 5). Graduate student Vikalp Raj of the Indian Institute of Science works in the field of solidstate batteries. He provided his artwork on lithium dendrites (see Figure 6), known to cause battery failure. "Beyond" Li-ion batteries is now a hot topic in materials research, and the MRS Meeting hosted a panel discussion on this subject.

\section{Spotlight on symposium sessions}

The 55 symposia were grouped into seven topical clusters: Broader Impact; Characterization and Modeling; Electronics and Optics; Energy and Sustainability; Nanoscale and Quantum Materials; Soft Materials and Biomaterials; and Structural Materials. One of the highlights was a panel discussion titled "Beyond Li-Ion Battery," featuring Nobel Laureate M. Stanley Whittingham of SUNY Binghamton, Shinichi Komaba of Tokyo University of Science, Christopher Johnson of Argonne National Laboratory, and Debra Rolison of the US Naval Research Laboratory. The panel discussion was moderated by symposium organizers Anton Van der Ven of the University of California, 


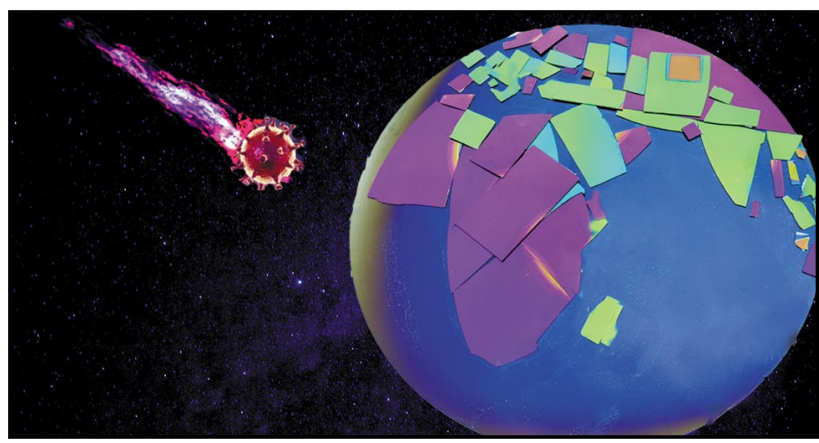

Figure 2. "The Silicon Planet vs Covid19 Comet," submitted by Poorani Gnanasambandan of the Luxembourg Institute of Science and Technology, won first place in the Science as Art competition. The image is a collage of thin films on $\mathrm{Si}$ deposited using atomic layer deposition. Blue: 12-in Si monitor consisting of a $91.84 \mathrm{~nm} \pm 2.32 \mathrm{~nm}$ MgO film. Pink: Wafer pieces with $201.24 \mathrm{~nm} \pm 1.28 \mathrm{~nm} \mathrm{ZnO}$ film.

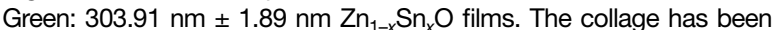
photoshopped onto the background. The background stars and COVID virus image are from Pixabay.

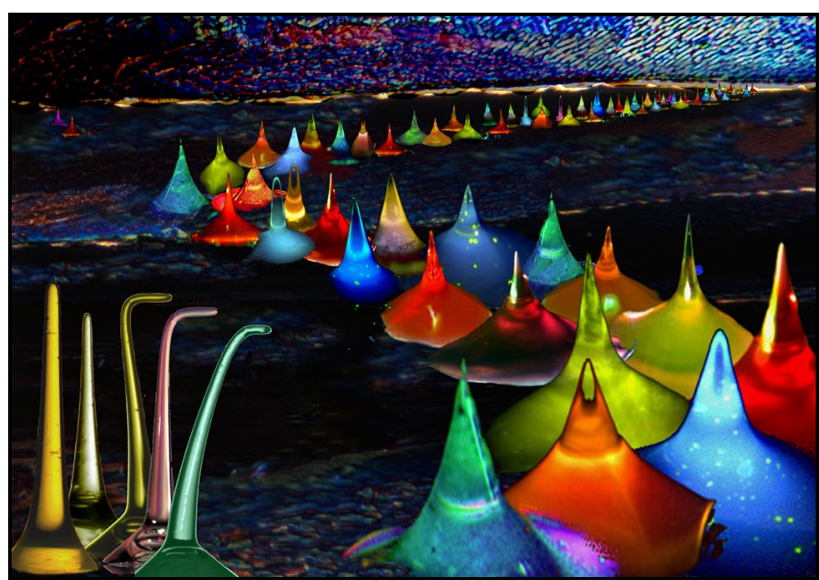

Figure 3. "La Marche des Aiguilles/The March of the Needles," submitted by Sara Coppola of CNR ISASI, won second place in the Science as Art competition. The artwork represents a collection of biocompatible and functionalized microneedles for drug delivery, just like the spectacular film La Marche de L'Empereur/ March of the Penguins (2005). The images were taken under photoluminescence emission of the microstructures fabricated by physical intelligence driven by the pyro-electric effect.

Santa Barbara, and Xin Li of Harvard University.

Having received the Nobel Prize for the development of the Li-ion battery, Whittingham opened the panel session with the current status of this type of battery and options and challenges to keeping it safe, also emphasizing that the development of batteries is very significant for solving problems due to climate change. He pointed out that the International Space Station replaced nickelmetal-hydride batteries with Li-ion batteries (LIBs) because the latter "last twice as long and they store the energy from the solar cells on the outside of the Space Station."

Whittingham believes Li intercalation systems will dominate for the next 5 to 10 years. He raised the question of using phosphates instead of layered oxides because they are safer, have lower costs, and present a stable cathode. While phosphates provide a low energy density (ED), he said this would be fine for most car applications.

$\mathrm{J}$ o h $\mathrm{n}$ s o $\mathrm{n}$ focused his presentation on $\mathrm{Si}$ anodes in LIBs, addressing the issue of how time factors into the loss in capacity. "We would like to control this rate," said Johnson, "So we're developing a predictive tool of a protocol to model and project the calendar lifetime." Battery development is happening so rapidly, he said, that his group wants to try to get this information to developers quickly.
LIBs with Si anodes are reaching very high energy densities but the target for the calendar life (the time in which the battery deteriorates whether used or not) is low at around 20 months, he said, when the current goal is 90 months or longer. Johnson's research group believes calendar aging is due to $\mathrm{Li}^{+}$ loss to the solid electrolyte interphase (SEI), which allows $\mathrm{Li}^{+}$transport. "An electrochemical assay that can determine the rate of charge consumption by the SEI can be used to screen electrode and electrolyte compositions that minimize calendar aging," he said.

Beyond LIBs, can sodium-, potassium-, or zinc-based systems find a market? Komaba said for $\mathrm{Na}$ and $\mathrm{K}$ batteries, "we have a good electrode ... but we still need much more deep investigation to conclude safety issues." A solid-state Na-ion battery is much more compact in size, he said. And $\mathrm{Na}$ and $\mathrm{K}$ chemistry for batteries show higher voltage than other elements. For example, Li-ion, $\mathrm{Na}$-ion, and $\mathrm{K}$-ion show $3+\mathrm{V}$ while $\mathrm{Mg}$ and $\mathrm{Ca}$ batteries are at $\sim 2 \mathrm{~V}$.

Rolison made the case for zinc-based batteries. "We definitely need a sustainable energy future and energy storage is critical to making that happen. And batteries will be a big chunk of what stores energy. So shouldn't the batteries themselves be sustainable?" she said.

Common batteries currently rely on high supply risk or scarce elements. Rolison said zinc-based batteries have a long successful history of use for military operations. They are low cost, use an aqueous electrolyte, and they are safe. The disadvantages, however, include very limited rechargeability, gassing from the aqueous electrolyte, and dendritic growth during cycling.

Rolison advocates for a new class of rechargeable $\mathrm{Zn}$-based batteries with a multielectron architected cathode, threedimensional (3D) redesign of the $\mathrm{Zn}$, and nanostructured electrolytes. She said the US Naval Research Laboratory is working on fabricating a 3D Zn "sponge" anode, which improves current distribution and suppresses dendrite formation.

Addressing the various battery technologies discussed, the moderators 


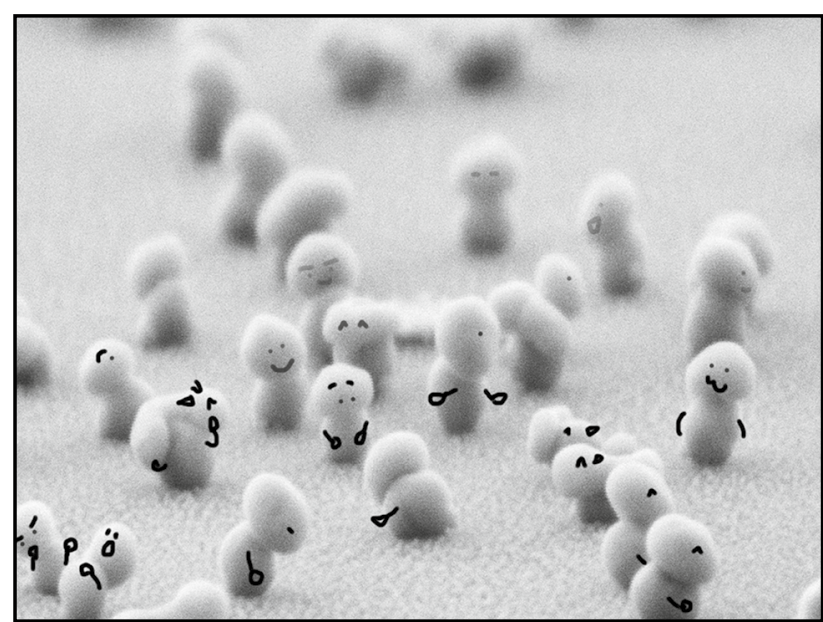

Figure 4. "Snowman Village," submitted by Eunjin Choi of the Max Planck Institute for Intelligent Systems, won second place in the Science as Art competition. A scanning electron microscope image of standing gold ( $\mathrm{Au}$ ) nanodimers separated by a dielectric nanogap. The snowman-like nanodimers are fabricated by a wafer-scale "lithography-free" scheme, using glancing angle deposition with thermal annealing.

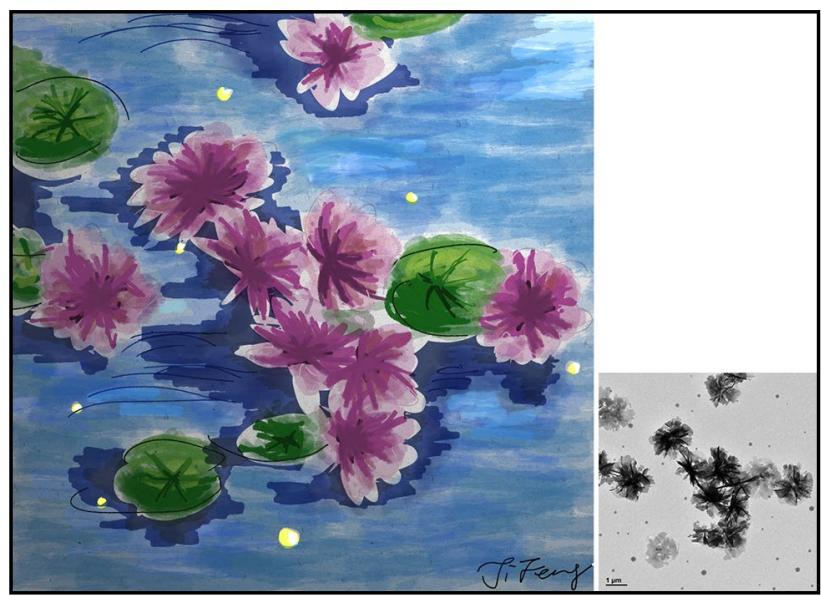

Figure 5. "Water Lilies in the Moonlight," submitted by Yadong Yin of the University of California, Riverside, won second place in the Science as Art competition. This artwork is inspired by a transmission electron microscope image of silicate nanosheet assemblies synthesized in a colloidal reaction. With a well-defined flower-like morphology, these nanosheet assemblies are reminiscent of water lilies blooming in a pond. The flowers and leaves were colored digitally using an oil painting effect, giving them vitality that bursts in tranquility and harmony.

asked the panelists about the biggest challenges to commercialization within the next few years. Komaba said that stationary applications should be possible for $\mathrm{Na}$ and $\mathrm{K}$ batteries because their structure is the same as for Li-ion batteries, which is "highly advantageous" for production. The safety issue is a problem with Li-ion batteries. "I believe that good electrolyte additives will be the key component and I think it was the one with the biggest challenge for $\mathrm{Na}$ and K batteries," said Komaba.

Whittingham said that when the electrolyte is oxidized, batteries are overcharged, "which is bad for the coulombic efficiency." $\mathrm{He}$ pointed out that the cathode does not get damaged, but the electrolyte should be non-catalytic.

Rolison said, "What I would like to see are the safety studies where we understand the rate and the depth of discharge and the number of cycles. You don't just assume a battery is safe because the primary is safe."

In the cluster of symposia on nanoscale and quantum materials, Dalia Chávez-García, director of the School of Engineering at CETYS Universidad, Mexico, presented her talk, "Luminescence Properties and Cell Uptake Analysis of Nanophosphors for Bio-imaging Applications." Nanoparticles are revolutionizing the field of biomedicine with their multivariate properties. Lately, up-conversion and down-conversion nanoparticles have received considerable focus as they possess easily controllable spectral characteristics that can be utilized in clinical applications such as deep-tissue bioimaging and nanomedicine. ChávezGarcía focused on using these optical nanomaterials as a diagnostic intervention to bioimage cancers.

Chávez-García's research group uses yttrium(III) oxide $\left(\mathrm{Y}_{2} \mathrm{O}_{3}\right)$ / gadolinium(III) oxide $\left(\mathrm{Gd}_{2} \mathrm{O}_{3}\right)$ and lanthanides (erbium/ytterbium) for the up-conversion nanoparticle synthesis. $\mathrm{Y}_{2} \mathrm{O}_{3}$ with europium or europium and bismuth are used for down-conversion nanoparticle synthesis. They are then functionalized with silica and amino groups to improve their biocompatibility, and then with folic acid to improve their specific targeting. Biocompatibility becomes critical for demonstrating the biolabeling capacity of the nanoparticles. Folic acid receptors are frequently overexpressed on cancer tissue, thus folic acid functionalization of nanoparticles promotes their internalization into the cancer cells specifically.

Characterization of the nanoparticles was performed by analyzing their photoluminescence spectra and transmission electron microscopy imaging to ensure that the luminescent emission and structure are maintained post-functionalization. Chávez-García reports that nanotoxicological tests show that the nanoparticles are non-cytotoxic, non-genotoxic, and hemocompatible against different cancer cell lines as well as nonmalignant cells. Confocal imaging and flow cytometry of the luminescent nanoparticles show that the nanoparticles were efficiently internalized into the cytoplasm of cancer cells. These results represent the up-conversion/down-conversion nanoparticles as a safe, biocompatible diagnostic biolabeling tool for cancer detection. 


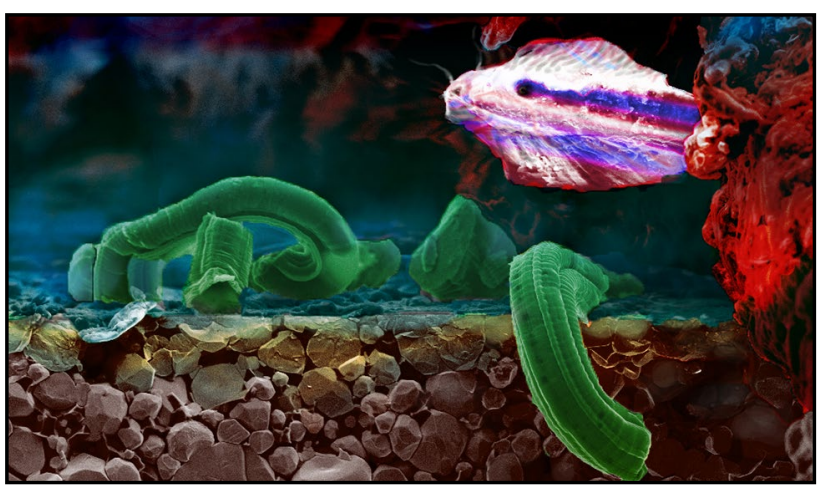

Figure 6. "Lithium Dendrite Aquarium," submitted by Vikalp Raj of the Indian Institute of Science, Bangalore, won first place in the Science as Art competition. This is a cross-sectional scanning electron microscope (SEM) image of a lithium/solid electrolyte $\left(\mathrm{Li}_{6.4} \mathrm{La}_{3} \mathrm{Zr}_{1.4} \mathrm{Ta}_{0.6} \mathrm{O}_{12}\right)$ interface. On the rightmost edge is a lithium metal interface with solid electrolyte (shaded in red). The fish is just a piece of lithium metal hanging from the parent lithium. The bottom rock-like morphology is the solid electrolyte. On top of the solid electrolyte is electrochemical growth of lithium dendrites (shaded in green) with a curvy rod-like morphology. The false color of the SEM image was applied through Adobe Photoshop. Photoshop credits: Sourayan Basu Bal.

A representative presentation in the cluster on Electronics and Optics was Ruth Shinar's talk on “Tunable Perovskite-Based Photodetectors in Optical Sensing." Compact spectroscopic platforms for (bio)chemical sensing have been proposed in the past. Typically, these consist of a light source in the visible range that sends an optical pulse to photoexcite a phosphorescent dye, which is embedded in a thin film like polystyrene. Once the visible light is turned off (end of the optical pulse), a signal of photoluminescence (at longer wavelength than the light source) is emitted from the dye and sensed by a photodetector (PD). Characteristics of the emitted signal (amplitude and decay time) depend on the interaction of the dye with the analyte (e.g., oxygen). As the analyte concentration increases, the amplitude of the photoluminescence signal is lower, and the decay time is shorter. Therefore, two measurement modes to determine the analyte concentration can be established by monitoring (1) the optical intensity or (2) decay time. The latter constrains the response time of the light source and the PD to be in the order of $1 \mathrm{~ms}$. Furthermore, it is desirable for the PD to have a low external quantum efficiency (EQE) at the wavelengths of the light source, but a high EQE at the wavelengths of the emitted signal of photoluminescence.

Shinar, a senior scientist at the Microelectronics Research Center and an adjunct professor in the Department of Electrical and Computer Engineering at Iowa State University, and her colleagues presented the assessment of various photodetection technologies. First, inorganic PDs based on inorganic materials such as amorphous silicon were evaluated making evident their long response time $(\sim 250 \mathrm{~ms})$ and the need for external filters. Second, organic PDs based on the polymer poly(3-hexylthiophene-2,5-diyl) or P3HT and the acceptor $[6,6]$-phenyl- $\mathrm{C}_{61}$-butyric acid methyl ester or PCBM were evaluated and showed low EQE in the region of interest. Finally, perovskite PDs were evaluated and showed a high EQE, fast response times, and the ability to have systems with broadband and narrowband photodetection sensitivity.

Alina Garcia Taormina, a National Defense Science and Engineering Graduate Research Fellow studying materials science at the University of Southern California, presented her work on magnetron sputtered microlattice structures in the cluster of symposia on Structural Materials. Architected lattice materials, which consist of a 3D periodic arrangement of struts, can be engineered on multiple length scales. As such, they hold many unique properties and functionalities, with applications ranging from battery electrodes to biomimetic scaffolds. However, a main challenge in the design of nano- and micro-architected systems is that current additive manufacturing techniques are restricted to polymer-based systems. To expand the synthesis space of such materials, coating techniques can be employed. Of particular interest is the use of magnetron sputtering, which allows for a wide variety of coating materials. "As the list of coating materials continues to grow, there exist vast opportunities for new coating material and lattice geometry combinations to be realized, potentially reaching new mechanical and functionality spaces," said Garcia Taormina.

Magnetron sputtering, however, is a momentum-driven line-of-sight process, resulting in large coating gradients, particularly around individual struts. This therefore necessitates a sputtering deposition that allows for increased line-of-sight coverage. To this end, Garcia Taormina reported the development of a hollow cathode magnetron sputtering approach for coating fine-featured lattice structures. Here, a hollow cylindrical target geometry is utilized instead of conventionally used planar cathodes. Such a configuration is advantageous as it allows for $360^{\circ}$ line-of-sight deposition instead of unidirectional deposition. To test this configuration, tetrahedral micro-architected lattices were coated using planar and hollow cathodes. Characterization studies demonstrated that compared to using a planar cathode, hollow cathode magnetron sputtering allowed for higher coating uniformity, with greater coating coverage on the outer struts and a thicker topside coating. These results demonstrate the potential of using hollow cathode magnetron sputtering as an approach to enhance line-of-sight sputtering and improved coating uniformity, opening new possibilities for the design of novel nano- and micro-architected lattice materials.

In the cluster of symposia on Characterization and Modeling, Anubhav Jain, staff scientist at Lawrence 
Berkeley National Laboratory, walked through the steps of "natural language processing" in his project called "MatScholar." Considering everyone has a huge stack of papers to read "some day" but can never get around to it, Jain's question: "Is there a way to use natural language processing, algorithms to actually, in some sense, read these papers for you and be able to give you information that you need from the research papers?"

One difficulty of looking up information on any given material, Jain explained, is due to the many ways chemical compositions are written. A search for "GaSb" will not match text that reads " $\mathrm{Ga}_{0.5} \mathrm{Sb}_{0.5}$ " A search for " $\mathrm{AgCrSe} \mathrm{C}_{2}$ " will not also suggest " $\mathrm{CuCrSe}{ }_{2}$ " as a similar result. It is also difficult to compile summaries such as all the known dopants in GaAs, said Jain.

"So we started a project called 'MatScholar,' an attempt to organize some of this information that exists in the research literature, focusing on things like application, synthesis methods, and characterization methods," said Jain. His research group is trying to accomplish this task by using machine learning.

For example, one of the projects is called "name entity recognition" that labels words by type, such as words that are materials $(\mathrm{GaN})$, descriptors (thin films), applications (laser diodes), synthesis methods, and properties. Over 4 million papers were collected, including abstracts and full text, from more than 2100 journals. So far, entities have been extracted from the abstracts, resulting in 31 million properties and 8.8 million characterization methods, for example. The entities were labeled manually for 1000 abstracts. The researchers then used an algorithm called "word embedding." This algorithm takes any word and turns it into a vector of numbers, said Jain. The researchers then use a neural network to try to predict which of these words might be materials and which ones might be simple descriptors. "The algorithm predicts context words around the target word," said Jain. "For materials knowledge, it's learning relationships, for example, between crystal structures."

The researchers tested the system from an earlier year to see if the suggestions have actually been studied in later years. They found that some of the suggestions have appeared in articles being published as the research team was developing this system. It seems, then, that this system can suggest what material might be good for an application.

Jain's group is also working on a doping database, data extraction from figures, and predicting the shapes of nanoparticles. Slides of Jain's presentation are posted at hackingmaterials. lbl.gov.

In the Broader Impact symposium, Kevin Jones, Distinguished Professor and Frederick N. Rhines Professor of Materials Science and Engineering at the University of Florida, described a one-credit honors course still under development in which he introduces the impact of materials science on sustainability. His first challenge is to teach students the concept of gigaton. "How much do we have to mitigate the $\mathrm{CO}_{2}$ level to actually have an impact on global warming," Jones said, teaching "the idea that we create 36 gigatons per year as a planet and 17 of it is too much." In addition, he needs to impress that the target keeps moving. "Everybody's standard of living is increasing and so, as the population increases and the standard of living increases, so does our $\mathrm{CO}_{2}$ production," said Jones. He illustrated this by having the students calculate what the excess would be if everybody in the world lived at the same standard of living as they do.

In order to break down this vast topic, Jones chose to use five categories that the US Environmental Protection Agency has identified as sources of carbon dioxide. The categories include electricity (which produces $49 \%$ of greenhouse gases), transportation $(20 \%)$, industry $(20 \%)$, commercial/residential $(9 \%)$, and agriculture (2\%) - according to the International Energy Agency via The World Bank, 2014.

Because so many materials are involved, Jones chose to teach about relatively large examples of renewables for electricity production and storage, including silicon for solar cells, new alloys for geothermal for production, and $\mathrm{Li}$-ion batteries and hydrogen for storage.

After teaching the course twice, Jones discovered that it is better to open the class with students finding articles on the topic to discuss, followed by lectures, instead of lecturing first. Since the students "introduce" themselves to the topic, the discussion about materials is less intimidating.

Jones said he found no appropriate textbook for the course, so he and David Ginley of the National Renewable Energy Laboratory in Colorado are developing an appropriate set of articles for the December 2021 issue of MRS Bulletin, which Jones will use as a supplement for the class. The theme is "Materials for the electrification of everything: Helping create a carbonneutral tomorrow." It will offer up-todate information. Jones also welcomes examples of fun materials innovations that will continue to keep the coursework moving with the times. He said the class could easily be expanded into a three-credit course.

For more news coverage of the scientific talks as well as MRS TV interviews and bloggers' impressions of the Meeting, go to the MRS Meeting Scene ${ }^{\circledR}$ blog at mrsmeetingscene.org.

The next MRS Meeting is the 2021 MRS Fall Meeting \& Exhibit, a hybrid event to be held in person in Boston, Mass., and online, November 28-December 3, 2021. 


\section{Thank you! to our 2021 Virtual MRS Spring Meeting Symposium Supporters!}

PLATINUM

Beijing Institute of Collaborative Innovation

National Science Foundation

GOLD

National Center for Advancing Translational Sci-

ences of the National Institutes of Health Grant
National Science Foundation

Science I AAAS

Swiss National Science Foundation

SILVER

Army Research Office
Helmholtz-Zentrum Berlin

IOP Publishing

JEOL Ltd.

Journal of Energy Chemistry

Taiyo Nippon Sanso

\section{M|R S OnDemand ${ }^{\circledR}$ WEBINAR SERIES}

An opportunity to learn about cuttingedge topics while networking with other researchers from around the world. Attendance is FREE.

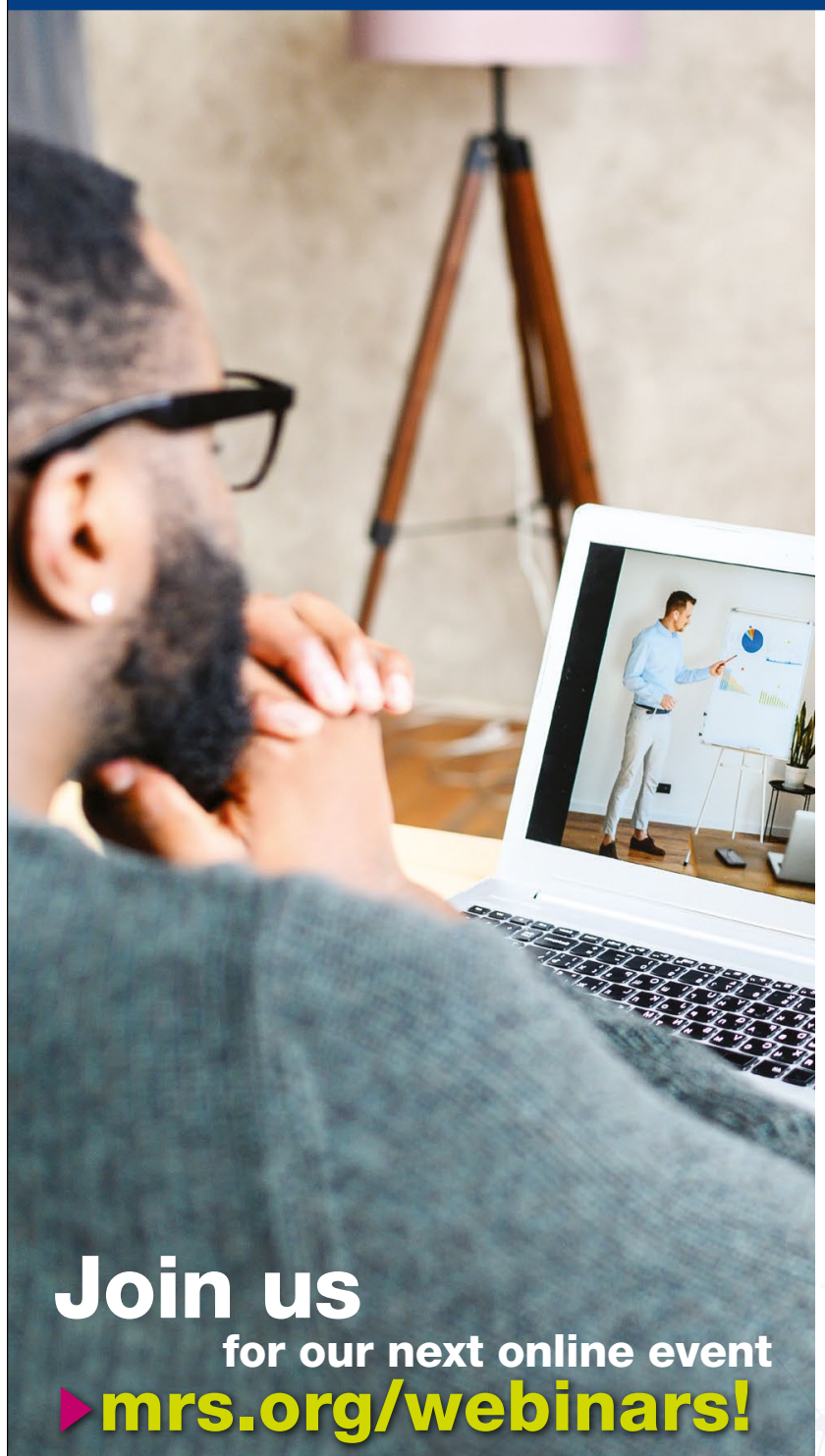

\section{UPCOMING WEBINARS}

- September 14, 2021

Materials Evolution in 3D/4D: Understanding Time-Evolved Processes Using Lab and Synchrotron X-ray Tomography Presented by Carl Zeiss Microscopy

\section{September 15, 2021}

Dynamic SIMS for Semiconductors - From compositional analysis of optoelectronics and 2D devices to implant depth-profiling of advanced Si-based structures Presented by CAMECA

\section{September 22, 2021}

Materials Development for the COVID-19 Pandemic

\section{September 28, 2021}

Learn from the Early Career Faculty Webinar Series Part II: Interview Process

\section{- September 29, 2021}

Green Cards for Scientific Researchers:

How to win your EB-1/NIW case

Presented by Getson \& Schatz

- October 20, 2021

Materials Opportunities for Low-Energy Computing

- October 26, 2021

Learn from the Early Career Faculty Webinar Series Part III: The Offer and Next Steps

- November 17, 2021

Oxide Electronics 